\title{
A transition-metal-free hydrogenation catalyst: Pore-confined sodium alanate for the hydrogenation of alkynes and alkenes
}

\author{
Peter L. Bramwell ${ }^{\text {, }}$ Jinbao Gao ${ }^{a}$, Bernd de Waal ${ }^{a}$, Krijn P. de Jong ${ }^{\text {a }}$, Robertus J.M. Klein Gebbink ${ }^{\mathrm{b}}$, \\ Petra E. de Jongh ${ }^{\mathrm{a}, *}$ \\ a Inorganic Chemistry and Catalysis, Debye Institute for Nanomaterials Science, Utrecht University, Universiteitsweg 99, 3583CG, The Netherlands \\ ${ }^{\mathrm{b}}$ Organic Chemistry and Catalysis, Debye Institute for Nanomaterials Science, Utrecht University, Universiteitsweg 99, 3583CG, The Netherlands
}

\section{A R T I C L E I N F O}

\section{Article history:}

Received 20 June 2016

Revised 25 August 2016

Accepted 19 September 2016

Available online 6 October 2016

\section{Keywords:}

Hydrogenation

Heterogeneous catalysis

Alkene

Alkyne

Sodium alanate

\begin{abstract}
A B S T R A C T
Hydrogenation catalysis is dominated by transition metals, but interest in alternative catalysts has been growing over recent years. Herein, a transition-metal-free catalyst is discussed consisting of carbon supported $\mathrm{NaAlH}_{4}$ as a selective catalyst for hydrogenation. This is illustrated using a range of substrates, and in more detail for the case of diphenylacetylene. Catalytic activity depends on the solvent utilized; in cyclohexane the activity is $2.3 \mathrm{~mol}(\mathrm{DPA}) \mathrm{mol}^{-1}\left(\mathrm{NaAlH}_{4}\right) \mathrm{h}^{-1}$ at $100 \mathrm{bar} \mathrm{H}_{2}, 150{ }^{\circ} \mathrm{C}$ with a slight preference for the formation of trans-stilbene. The catalyst selectivity is influenced by the loading, yielding a high selectivity toward the thermodynamically less stable cis-stilbene at low catalyst loadings. This proof of principle shows promise for using metal hydrides based on earth-abundant elements as effective hydrogenation catalysts.
\end{abstract}

(c) 2016 Elsevier Inc. All rights reserved.

\section{Introduction}

Hydrogenation of C-C multiple bonds is a key step in the synthesis of many important compounds [1-3]. As with many other fields of catalysis, transition metals dominate. For example, Pd is widely employed in hydrogenation of alkynes to alkenes [4-8], particularly in the case of the famous Lindlar catalyst [9]. Pdbased catalysts generally show very high activity (turnover frequencies, TOF, of $2500 \mathrm{~h}^{-1}$ at $22{ }^{\circ} \mathrm{C}$ ) [10] under mild conditions but often relatively low selectivities $[11,12]$.

In recent years a new family of transition-metal-free catalysts, frustrated Lewis pairs, has emerged which boast reasonably high activities (TOFs of $5-40 \mathrm{~h}^{-1}$ at $80^{\circ} \mathrm{C}$ ) [13] and selectivities toward cis-alkenes without utilizing transition metals [14,15]. Light metal hydrides such as $\mathrm{LiAlH}_{4}$ and $\mathrm{NaBH}_{4}$ are based on earth-abundant elements and are typically used in the stoichiometric reduction of polar groups such as carbonyls $[16,17]$. However, these materials so far have seldom been applied to the reduction of alkenes or alkynes and in such cases only as stoichiometric reagents, rather

\footnotetext{
Abbreviations: XRD, X-ray diffraction; SEM, scanning electron microscopy; EDX, energy dispersive X-ray spectroscopy; TPD, temperature programmed desorption; TCD, thermal conductivity detector; DPA, diphenylacetylene; PEG, polyethylene glycol; GC, gas chromatography.

* Corresponding author.

E-mail address: P.E.dejongh@uu.nl (P.E. de Jongh).
}

than as catalysts. There is one isolated example of $\mathrm{NaAlH}_{4}$ being utilized as a hydrogenation catalyst where $\mathrm{Ti}$ and $\mathrm{NaAlH}_{4}$ were milled together and tested in the hydrogenation of diphenylacetylene [18]. At $130^{\circ} \mathrm{C}$ and 100 bar of hydrogen pressure the alkyne starting material was fully hydrogenated to the saturated product within three hours. Ti-doped sodium alanate is known to reversibly store and release hydrogen, which might explain its catalytic activity.

The preparation and characterization of confined light metal hydrides for the purpose of reversible uptake and release of hydrogen (hydrogen storage) at reasonably low temperatures with fast kinetics have been demonstrated in recent years [19-27]. Melt infiltration under hydrogen pressure (to prevent decomposition) can be utilized to prepare these materials. The metal hydride and support are mixed together and heated to the melting point of the hydride. The molten hydride infiltrates the pores and, upon cooling, produces solid metal hydride confined within the pores, which is referred to as nanoconfinement. The resulting material consists of a carbon matrix of which the pores are filled with metal hydride. This pore-confined metal hydride lacks long range crystallinity and demonstrates orders of magnitude faster kinetics of absorption and desorption compared to the corresponding macrocrystalline metal hydride material.

In the case of $\mathrm{NaAlH}_{4}$ almost full reversibility of hydrogen release and uptake was achieved at $150^{\circ} \mathrm{C}$ [21]. Reduction of the 
particle size improves the kinetics of hydrogen sorption as bulk crystalline $\mathrm{NaAlH}_{4}$ displays much slower uptake kinetics and requires temperatures above $180^{\circ} \mathrm{C}$ to desorb hydrogen. Additionally, the presence of carbon alters the hydrogen release profile from a three-step pathway to a two-step pathway, bypassing the intermediate $\mathrm{Na}_{3} \mathrm{AlH}_{6}$ phase altogether [21]. Nanoconfinement promotes reversibility by preventing the macroscopic phase separation of $\mathrm{NaH}$ and Al. All of these factors allow the $\mathrm{NaAlH}_{4} / \mathrm{C}$ nanocomposite to release and reabsorb hydrogen in a reversible manner at relatively low temperatures. This raises the interesting question of whether this material could also be used as a hydrogenation catalyst in the absence of any transition metals [18,28]. Hence we demonstrate carbon-confined $\mathrm{NaAlH}_{4}$, previously developed for hydrogen storage purposes, as a transition-metal-free hydrogenation catalyst. We explore the hydrogenation of alkynes and alkenes, while studying in detail the recyclability, selectivity, and activity depending on solvent and catalyst loading in the case of DPA hydrogenation (Scheme 1).

\section{Experimental section}

All materials were stored in a nitrogen-filled glove box (Mbraun Labmaster I30, $1 \mathrm{ppm} \mathrm{H}_{2} \mathrm{O},<1 \mathrm{ppm} \mathrm{O}_{2}$ ) prior to use, except for the catalyst which was stored in an argon-filled glove box (Mbraun Labmaster dp, 1 ppm $\mathrm{H}_{2} \mathrm{O},<1 \mathrm{ppm} \mathrm{O}_{2}$ ) and transferred to the other glove box immediately before use. $\mathrm{NaAlH}_{4}$ powder (hydrogen storage grade), DPA (98\%), cyclohexane (anhydrous, 99.5\%), dodecane (anhydrous, $\geqslant 99 \%$ ), 1-octyne (97\%), 4-octyne (99\%) and styrene (99\%) were all obtained from Sigma-Aldrich. Toluene was dried in a distillation apparatus before storage. All liquid reagents and solvents were stored in the glove box over molecular sieves following degassing by bubbling nitrogen gas through the liquid for several hours. DPA was dried in vacuo overnight before storage in the glove box. The carbon aerogel was prepared by the sol-gel resorcinol procedure [26] and was analyzed by nitrogen physisorption (performed at $-196{ }^{\circ} \mathrm{C}$, Micromeritics TriStar) to determine the pore characteristics (BET surface area $564 \mathrm{~m}^{2} \mathrm{~g}^{-1}$, pore volume $0.57 \mathrm{~cm}^{3} \mathrm{~g}^{-1}$, broad pore size distribution with a maximum around $18-20 \mathrm{~nm})$. The aerogel was dried at $600{ }^{\circ} \mathrm{C}$ under argon flow for $12 \mathrm{~h}$ before storing in the argon-filled glove box.

The catalyst was prepared by melt infiltration according to previously reported literature [21] with a loading of $20 \mathrm{wt} \% \mathrm{NaAlH}_{4}$. The result was analyzed by X-ray Diffraction (Bruker AXS D8 advance 120 machine, Co-K $\mathrm{K}_{\alpha}$ radiation, air-tight sample holder used), Temperature Programmed Desorption (Micromeritics AutoChem II, equipped with a TCD detector, Ar flow of $25 \mathrm{~mL} \mathrm{~min}^{-1}$ ) and nitrogen physisorption before use in catalytic tests. Multiple batches of the catalyst were prepared using the above procedure and their full characterization can be found in Section S1 of the supporting information.

All hydrogenation reactions were carried out in a Parr $300 \mathrm{~mL}$ autoclave at $150^{\circ} \mathrm{C}, 100$ bar hydrogen pressure and constant stirring. The autoclave was loaded in the nitrogen glove box. $270 \mathrm{mg}$ of catalyst $\left(1 \mathrm{mmol} \mathrm{NaAlH}_{4}\right)$ and $890 \mathrm{mg}$ of DPA ( $5 \mathrm{mmol}$ ) were added to $180 \mathrm{~mL}$ of solvent. Dodecane was added as an internal standard. Aliquots of reaction mixture were taken for analysis at regular intervals using a sampling arm which was flushed with a small amount of reaction mixture before the taking of each sample (roughly $2 \mathrm{~mL}$ of reaction mixture per sample). Samples were then analyzed by Gas Chromatography (GC-2010 Shimadzu gas chromatograph equipped with a Shimadzu AOC-20i Auto injector) using a CP-Wax column to separate the reaction mixture by boiling points; Boiling points: DPA $\left(300^{\circ} \mathrm{C}\right)$, cis-stilbene $\left(307^{\circ} \mathrm{C}\right)$, transstilbene $\left(305^{\circ} \mathrm{C}\right)$, bibenzyl $\left(284^{\circ} \mathrm{C}\right)$.

The following blank and reference measurements were performed, the results of which can be found in Sections S2-S3 in the supporting information. The influence of the carbon support on the activity was tested by performing the above catalysis experiments using only $215 \mathrm{mg}$ of the carbon support in the absence of $\mathrm{NaAlH}_{4}$. To rule out the effect of the metals in the autoclave walls catalytic tests were performed using a Teflon lining in the autoclave. As the Teflon liner reduced the volume of the reaction vessel the reaction was scaled down by $15 \%$. The catalytic tests were repeated to demonstrate reproducibility. The recyclability of the catalyst was tested by recovering the catalyst at the end of the hydrogenation reaction and re-using in another test. Since $150 \mathrm{mg}$ of the $\mathrm{NaAlH}_{4} / \mathrm{C}$ catalyst was recovered (due to losses through filtering and removing the catalyst from the reactor) the catalytic loading in the second reaction was $150 \mathrm{mg}$ instead of $270 \mathrm{mg}$. Isomerization studies were carried out by performing the reaction in the same manner as above but instead of adding DPA either trans-stilbene or cis-stilbene was added.

For the experiments on the adsorption of each substrate on the catalyst in different solvents the following procedure was applied. All experiments were performed at room temperature to ensure no hydrogenation reaction occurred. In each experiment a substrate, either DPA, cis-stilbene, trans-stilbene or bibenzyl, was dissolved in $9 \mathrm{~mL}$ of the solvent and stirred for $5 \mathrm{~min}$. This was then analyzed by gas chromatography to determine the substrate concentration at $100 \%$. Then $100 \mathrm{mg}$ of catalyst was added (with a volumetric liquid:solid ratio of 203) to the solution and a sample was taken at 60 min for gas chromatography (GC) analysis. The decrease in

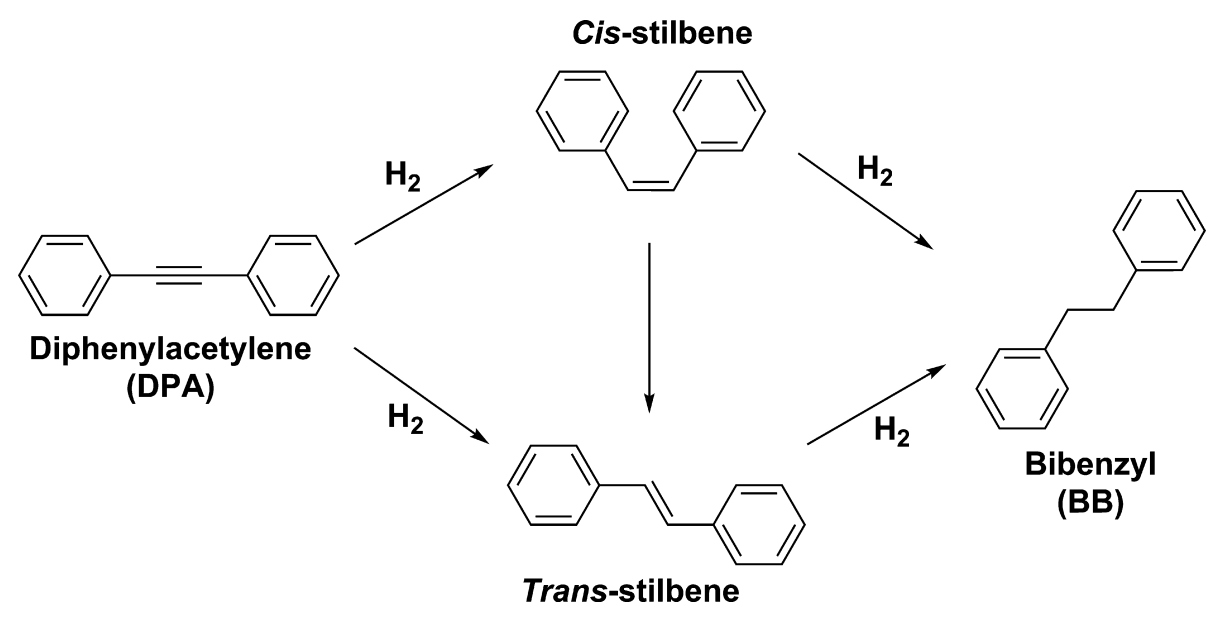

Scheme 1. Reaction scheme showing the hydrogenation of diphenylacetylene to bibenzyl. 
concentration was assumed to be due to substrate adsorption on the catalyst surface, allowing the calculation of the quantity of adsorbed substrate. Each sample was run in the GC three times and an average was taken, yielding the points shown in Fig. S6.1, and the variation in these three measurements was then used to calculate the error given in Table 2.

\section{Results and discussion}

\subsection{Catalyst characterization}

The catalyst is prepared by melt infiltration of $\mathrm{NaAlH}_{4}$ into the pore structure of a carbon aerogel support (volume average pore diameter of $13 \mathrm{~nm}$, prepared by the sol-gel resorcinol method) [29]. A schematic of the melt infiltration procedure is shown in Fig. 1. Following melt infiltration X-ray diffraction (XRD) shows the loss of crystalline $\mathrm{NaAlH}_{4}$ and a small amount of crystalline $\mathrm{Al}$ is present. $\mathrm{Al}$ is present due to decomposition of a minor fraction of the $\mathrm{NaAlH}_{4}$ to $\mathrm{NaH}$ and Al during melt infiltration [21]. The low intensity of the $\mathrm{NaAlH}_{4}$ signal shows that pore-confined $\mathrm{NaAlH}_{4}$ lacks long range crystallinity, where the proportion of crystalline $\mathrm{NaAlH}_{4}$ is $29 \%$ compared to the physical mixture, indicating a confinement of $71 \%$ of the $\mathrm{NaAlH}_{4}$. This is supported by the pore volume loss upon melt infiltration of $26 \mathrm{vol} \%$ ( $\pm 2 \mathrm{vol} \%$ ), which accounts for $91 \%$ of the $\mathrm{NaAlH}_{4}$ present. Mapping of the distribution of $\mathrm{NaAlH}_{4}$ and $\mathrm{Al}$ through the catalyst surface has been well studied previously through use of scanning electron microscopy (SEM) and energy dispersive X-ray spectroscopy (EDX) [21]. As a result it is known that the $\mathrm{NaAlH}_{4}$ is distributed in a homogeneous manner. Hence the catalyst does not contain discrete $\mathrm{NaAlH}_{4}$ particles but rather the pores are either completely filled or empty, with $\mathrm{NaAlH}_{4}$ exposed at the pore mouths at the external surface of the carbon support particle, and at the boundaries between filled and unfilled pore regions.

Characterization of the hydrogen content of nanoconfined hydride materials is commonly performed using temperature programmed desorption (TPD) and the temperature at which the hydrogen can be released is also relevant to hydrogenation catalysis. In the $\mathrm{NaAlH}_{4} / \mathrm{C}$ nanocomposite hydrogen release (Fig. 2) begins at $100{ }^{\circ} \mathrm{C}$ with a maximum release rate at $175^{\circ} \mathrm{C}$ and a total emission of $0.37 \mathrm{wt} \%$ of hydrogen (compared to a theoretical capacity of $1.48 \mathrm{wt} \%$ ). This hydrogen release should be reversible

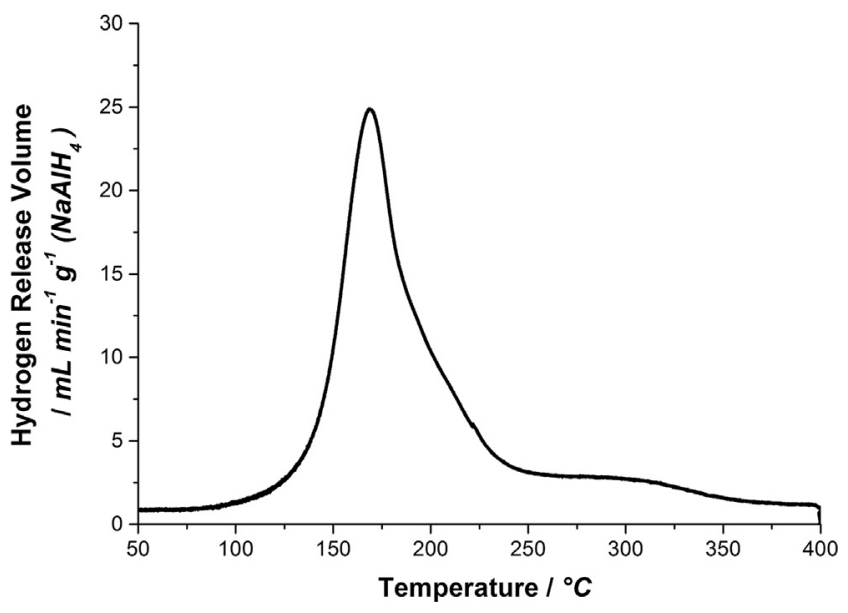

Fig. 2. Temperature programmed desorption profile showing the hydrogen release of the $\mathrm{NaAlH}_{4} / \mathrm{C}$ catalyst performed at a heating rate of $5^{\circ} \mathrm{C} \mathrm{min}^{-1}$ under $25 \mathrm{~mL} \mathrm{~min}^{-1}$ Ar flow.

in order for $\mathrm{NaAlH}_{4}$ to perform hydrogenation reactions in a catalytic manner and full reversibility at $150{ }^{\circ} \mathrm{C}$ has indeed been demonstrated in the literature [20], where the optimal reversible hydrogen capacity is achieved with rehydrogenation at 100 bar of $\mathrm{H}_{2}$. Therefore a temperature of $150{ }^{\circ} \mathrm{C}$ was used for all catalytic testing.

\subsection{Catalyst testing}

Fig. 3 shows the hydrogenation of DPA performed using the $\mathrm{NaAlH}_{4} / \mathrm{C}$ catalyst under 100 bar $\mathrm{H}_{2}$ of pressure. It is clear that the nanocomposite exhibits catalytic behavior by fully hydrogenating DPA when used in a 5:1 DPA: $\mathrm{NaAlH}_{4}$ molar ratio (turnover number 10 ) with a molar activity, taken between $90 \%$ and $50 \%$ DPA conversion, of $2.29 \mathrm{~mol}(\mathrm{DPA}) \mathrm{mol}^{-1}\left(\mathrm{NaAlH}_{4}\right) \mathrm{h}^{-1}$. Due to the nature of the catalyst it is difficult to estimate a turnover frequency (TOF) as it is not possible to measure the amount of exposed active surface area. Only a small amount of the $\mathrm{NaAlH}_{4}$ in the catalyst is active, as it is confined within the pores; therefore, for this catalyst a large fraction of the $\mathrm{NaAlH}_{4}$ is not exposed to the reactants. For this reason a TOF range has been calculated with a

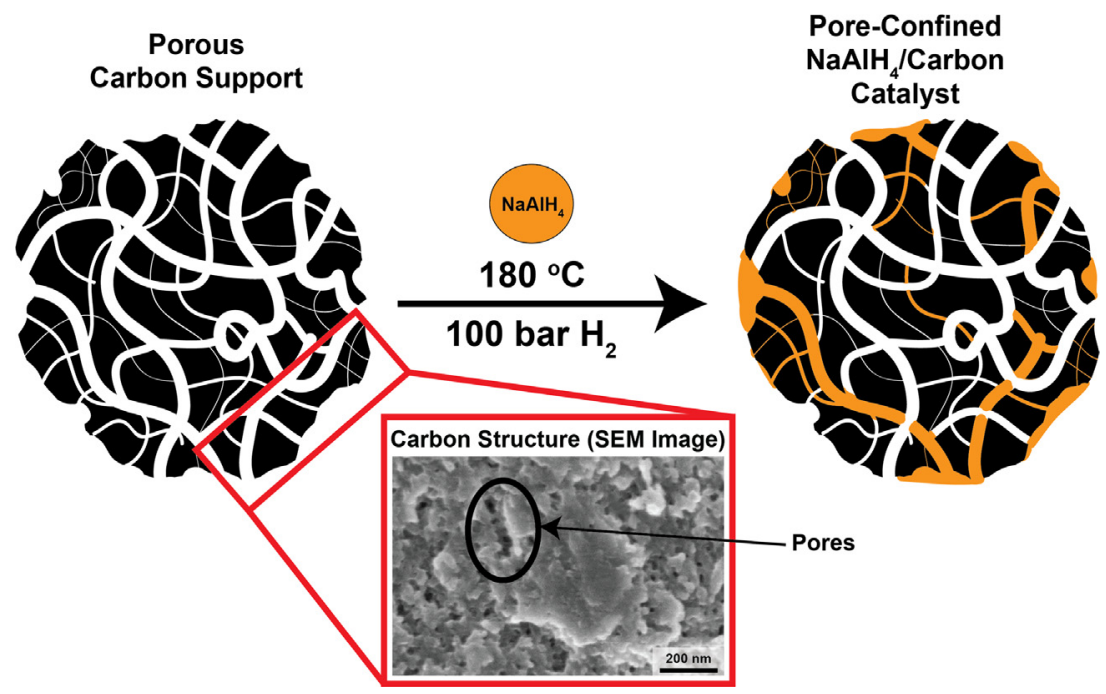

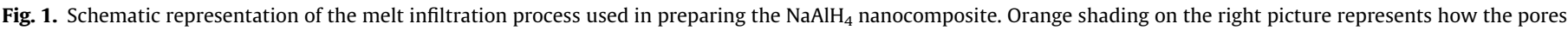
are filled by $\mathrm{NaAlH}_{4}$. (For interpretation of the references to color in this figure legend, the reader is referred to the web version of this article.) 


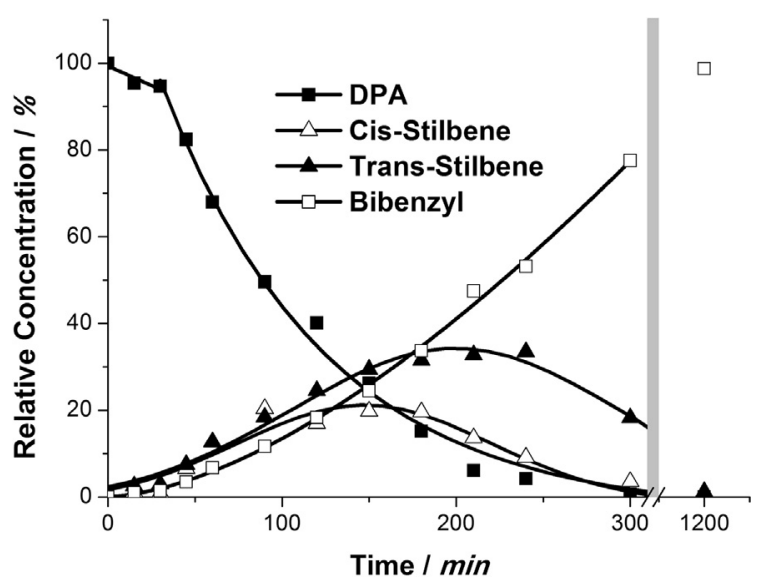

Fig. 3. Concentrations during hydrogenation of DPA performed with cyclohexane as a solvent. Reaction performed under $100 \mathrm{bar} \mathrm{H}_{2}$ pressure, at $150{ }^{\circ} \mathrm{C}$, with a catalyst loading of $270 \mathrm{mg}\left(1 \mathrm{mmol}\right.$ of $\left.\mathrm{NaAlH}_{4}\right)$ and $5 \mathrm{mmol}$ of DPA.

lower limit and a more realistic estimate. The lower limit is based on the assumption that all of the BET surface areas measured by nitrogen physisorption are composed of available catalytically active sites. A more realistic estimation was made by using the volume fraction of $\mathrm{NaAlH}_{4}$ in the catalyst, and assuming that the fraction of the total surface area consisting of $\mathrm{NaAlH}_{4}$ was the same as the volume fraction of $\mathrm{NaAlH}_{4}$ (see supporting information Section S4 for further details). The turnover frequency has been estimated to be 2 or $179 \mathrm{~h}^{-1}$ for the lower and more realistic estimate respectively. Although significantly lower than a typical Pd-based catalyst, these values are comparable to those for Frustrated Lewis Pair catalysts [13]. The alkyne functionality in the DPA is fully reduced to the alkane (bibenzyl) by the catalyst. This demonstrates that the catalyst can in fact hydrogenate both alkynes and alkenes.

Fig. 4 shows the dependence of the rate of the DPA consumption on catalyst loading. Each point was generated by performing the reaction for $2 \mathrm{~h}$ with samples being taken every $10 \mathrm{~min}$ for GC analysis. In this way the rate of DPA consumption up to $50 \%$ was determined accurately for each catalyst loading. The rate increases linearly with catalyst loading. Since there is a small amount of DPA conversion in the absence of catalyst it is possible that the metals in the walls of the autoclave, in which the reaction is carried out,

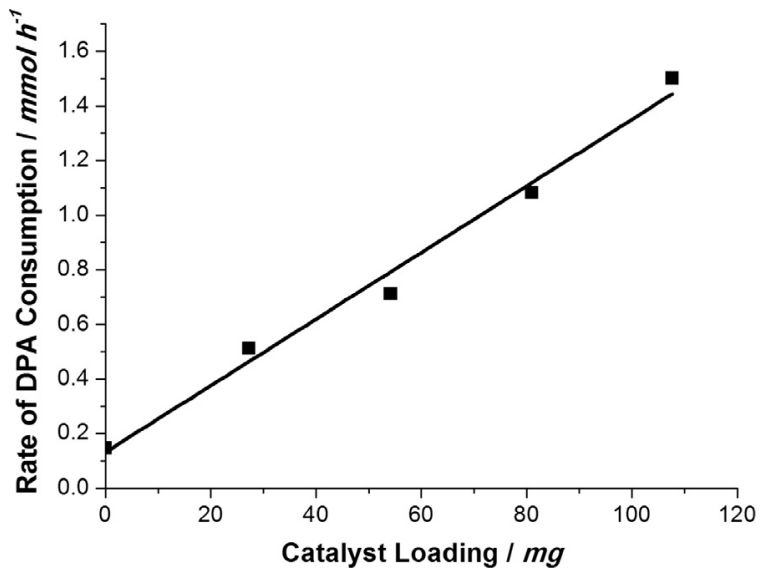

Fig. 4. Average rate of DPA hydrogenation versus catalyst loading in cyclohexane. Each data point was determined from the time taken to reach $50 \%$ conversion of DPA. All experiments were performed at $150^{\circ} \mathrm{C}, 100$ bar $\mathrm{H}_{2}$ with GC samples taken every $10 \mathrm{~min}$ for $2 \mathrm{~h}$. are at least partially responsible for the observed activity of the $\mathrm{NaAlH}_{4} / \mathrm{C}$ catalyst. To rule this out the reaction was also performed with a Teflon insert in the autoclave (Fig. S2.1). When the Teflon liner is used the activity is $2.32 \mathrm{~mol}(\mathrm{DPA}) \mathrm{mol}^{-1}\left(\mathrm{NaAlH}_{4}\right) \mathrm{h}^{-1}$, which is only a $1 \%$ deviation from the activity seen when the Teflon insert is not used. Further, the carbon support itself showed no effect on the rate of DPA hydrogenation (see supporting information Section S2). Therefore it can be concluded that the metals in the autoclave walls and the carbon support are not the source of the apparent catalytic activity of the $\mathrm{NaAlH}_{4} / \mathrm{C}$ catalyst.

To determine recyclability of the catalyst a reaction was performed at $150{ }^{\circ} \mathrm{C}, 100$ bar hydrogen pressure in a cyclohexane solvent with a catalyst loading of $270 \mathrm{mg}$ and DPA loading of $5 \mathrm{mmol}$. After the reaction was finished the catalyst was recovered by filtration, dried and used again in a hydrogenation reaction under the same conditions (Fig. 5). In the second run $150 \mathrm{mg}$ of the catalyst was recovered so the amount of catalyst was $44 \%$ lower. This is reflected in the data as the rate of hydrogenation is $53 \%$ slower in the second run.

In order to further determine the scope of the catalyst a number of other substrates were also tested under the same conditions (Table 1, see supporting information Section S5 for reaction profiles). 1-octyne and 4-octyne were chosen as they are unfunctionalized alkynes and can demonstrate whether the catalyst is able to reduce terminal and internal alkynes respectively. Here it is clear that the catalyst can effectively reduce the unfunctionalized molecules and is more effective in reducing internal alkynes than terminal alkynes. This is also true for the resulting alkenes, which are further reduced to alkanes, demonstrating the ability of the catalyst to also reduce unfunctionalized alkenes. Hydrogenation of a conjugated alkene, styrene, further demonstrates the wide scope of the applicability of the catalyst.

\subsection{Solvent effects}

In previous literature [27] the hydrogenation of DPA was performed in toluene, and for this reason toluene was also used as a solvent for comparison to cyclohexane (Fig. 6). In toluene $50 \%$ conversion of DPA is achieved in roughly $350 \mathrm{~min}$ (activity of $0.48 \mathrm{~mol}$ (DPA) $\left.\mathrm{mol}^{-1}\left(\mathrm{NaAlH}_{4}\right) \mathrm{h}^{-1}\right)$ whereas the same conversion when cyclohexane is employed occurs around $90 \mathrm{~min}$ (2.29 mol (DPA) $\left.\mathrm{mol}^{-1}\left(\mathrm{NaAlH}_{4}\right) \mathrm{h}^{-1}\right)$, which is comparable to that for a Ti-doped ball-milled $\mathrm{NaAlH}_{4}$ catalyst under similar conditions.

The solvent-dependence of the activity is striking and may be a result of either differences in hydrogen solubility or interaction with the catalyst. At room temperature the solubility of hydrogen in cyclohexane is 35\% higher than for toluene at a pressure of 44 bar [30], but this can only account for a small portion of the difference in activity. Therefore it is very likely that something other than hydrogen solubility is affecting activity, such as competitive adsorption from the solvent. To verify this hypothesis we performed experiments on the adsorption of each substrate onto the catalyst surface in both solvents (Table 2. Details of the experiments and calculations can be found in S6 of the supporting information). They showed that there is a higher level of substrate adsorption in cyclohexane compared with toluene. Therefore, it can be inferred that toluene strongly adsorbs onto the catalyst surface, hindering the adsorption and subsequent reaction of DPA.

\subsection{Selectivity control}

The catalyst favors the production of trans-stilbene, which is not surprising as trans-stilbene is the thermodynamically more stable isomer [31]. There are two possible explanations for this, either this is the intrinsic selectivity of the catalyst or isomerization of cis-stilbene to trans-stilbene during reaction is affecting the 

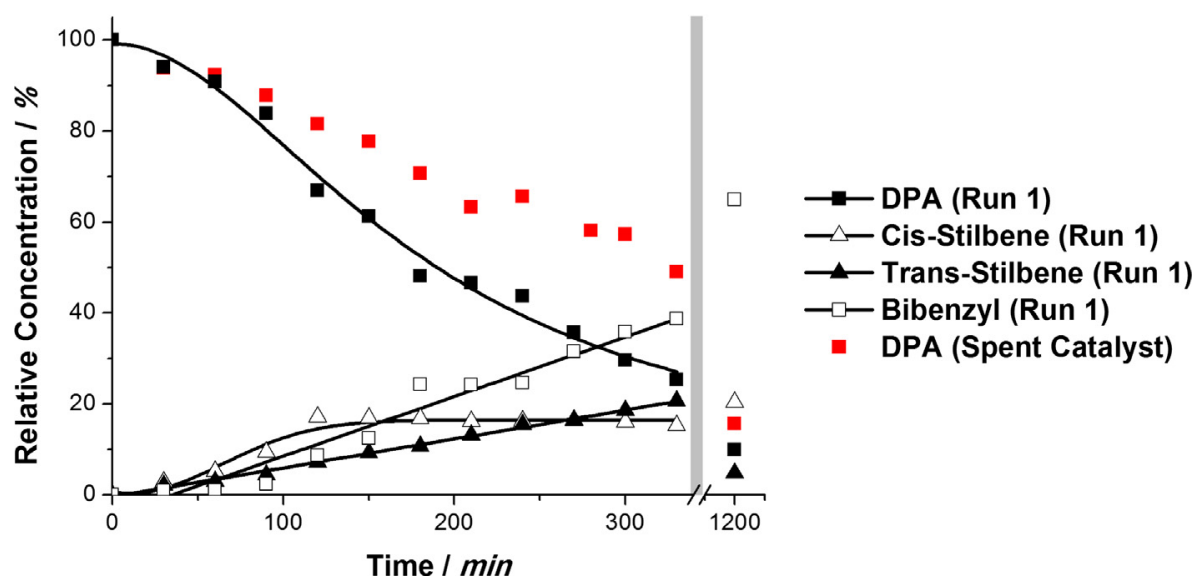

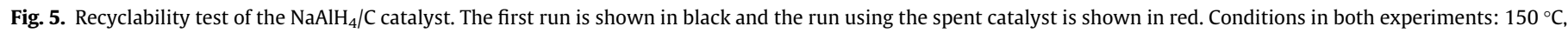

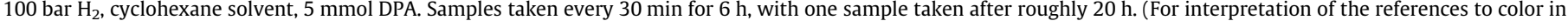
this figure legend, the reader is referred to the web version of this article.)

Table 1

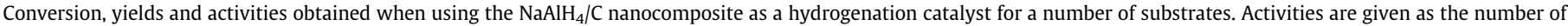
moles of hydrogenated substrate per hour, normalized to the total number of moles of $\mathrm{NaAlH}_{4}$ in the $\mathrm{NaAlH}_{4} / \mathrm{C}$ catalyst (roughly 1 mmol).

\begin{tabular}{|c|c|c|c|c|}
\hline Substrate $^{\mathrm{a}}$ & Conversion/\% & Activity & Product & Yield/\% \\
\hline \multirow[t]{3}{*}{ DPA } & 100 & 1.00 & Cis-stilbene & 0 \\
\hline & & & Trans-stilbene & 0 \\
\hline & & & Bibenzyl & 100 \\
\hline \multirow[t]{2}{*}{ 1-Octyne } & 100 & 0.19 & 1-Octene & 47 \\
\hline & & & Octane & 43 \\
\hline \multirow[t]{2}{*}{ 4-Octyne } & 95 & 0.23 & 4-Octene & 2 \\
\hline & & & Octane & 93 \\
\hline Styrene & 97 & 0.21 & Ethylbenzene & 97 \\
\hline
\end{tabular}

a Reactions performed at $150{ }^{\circ} \mathrm{C}, 100$ bar $\mathrm{H}_{2}$ pressure for $48 \mathrm{~h}$ in a cyclohexane solvent, $\mathrm{NaAlH}_{4}$ :substrate molar ratio of $1: 5$.

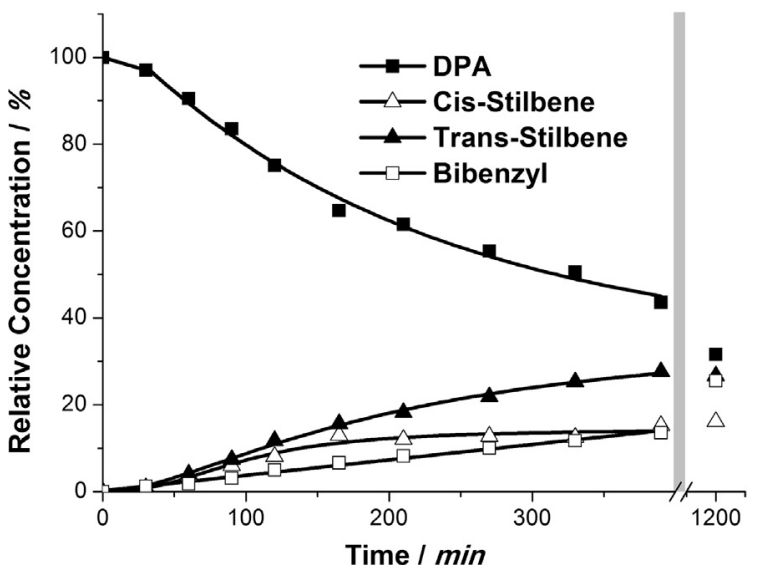

Fig. 6. Hydrogenation of DPA performed with toluene as a solvent. Each data point corresponds to a sample taken at the respective time and analyzed by gas chromatography. Reactions performed under 100 bar $\mathrm{H}_{2}$ pressure, at $150{ }^{\circ} \mathrm{C}$, with a catalyst loading of $270 \mathrm{mg}\left(1 \mathrm{mmol}\right.$ of $\left.\mathrm{NaAlH}_{4}\right)$ and $5 \mathrm{mmol}$ of DPA.

observed selectivity. To verify this we monitored two separate reactions: hydrogenation of trans-stilbene and hydrogenation of cis-stilbene rather than DPA (Fig. 7). It was found that the rate of trans-stilbene formation from cis-stilbene is roughly $1.2 \mathrm{mmol} \mathrm{h}^{-1}$ whereas isomerization from trans- to cis-stilbene does not occur at all. This rate of isomerization can account for $100 \%$ of the transstilbene that is formed during reaction. Therefore, the catalyst primarily produces cis-stilbene through hydrogenation of DPA and trans-stilbene is only formed through isomerization of the cisstilbene product.
Table 2

Summary of adsorption data for each substrate in cyclohexane and toluene.

\begin{tabular}{llll}
\hline Solvent & Substrate & $\begin{array}{l}\text { Concentration drop } \\
\text { after } 60 \mathrm{~min} / \%\end{array}$ & $\begin{array}{l}\text { \% of catalyst } \\
\text { surface coverage }\end{array}$ \\
\hline Cyclohexane & DPA & 17.8 & $3.82 \pm 0.03$ \\
& Cis-stilbene & 16.0 & $4.04 \pm 0.04$ \\
& Trans-stilbene & 15.9 & $3.38 \pm 0.02$ \\
Toluene & Bibenzyl & 10.8 & $2.79 \pm 0.03$ \\
& DPA & 1.5 & $0.35 \pm 0.00(2)$ \\
& Cis-stilbene & 1.9 & $0.82 \pm 0.01$ \\
& Trans-stilbene & 3.4 & $0.61 \pm 0.00(4)$ \\
& Bibenzyl & 0.5 & $0.10 \pm 0.00(03)$
\end{tabular}

a Calculated from the BET surface area of the catalyst.

An interesting question given that isomerization of cis-stilbene can take place at the free surface of the catalyst is whether the number of active sites can affect the selectivity. Therefore a high molar DPA: $\mathrm{NaAlH}_{4}$ ratio of 25:1 was applied, by reducing the catalyst loading (Fig. 8). The lower loading drastically altered the selectivity, yielding a cis:trans ratio of 12 at $50 \%$ conversion compared to a ratio of 1 at the same conversion when the lower DPA: $\mathrm{NaAlH}_{4}$ ratio of 5 is used. The reason for this is possibly blocking of the active site required for isomerization by DPA at lower catalyst loadings. In any case the fact that the catalyst loading has such a marked impact on the selectivity demonstrates that this is not thermal isomerization. Compared to the more active Pd catalysts this provides the unique opportunity to tune selectivity by changing catalyst loading.

In order to gain more insight into the mode of action of the catalyst, particularly whether hydrogen can be directly extracted from the $\mathrm{NaAlH}_{4}$ lattice or whether it is provided by the gas phase only, 

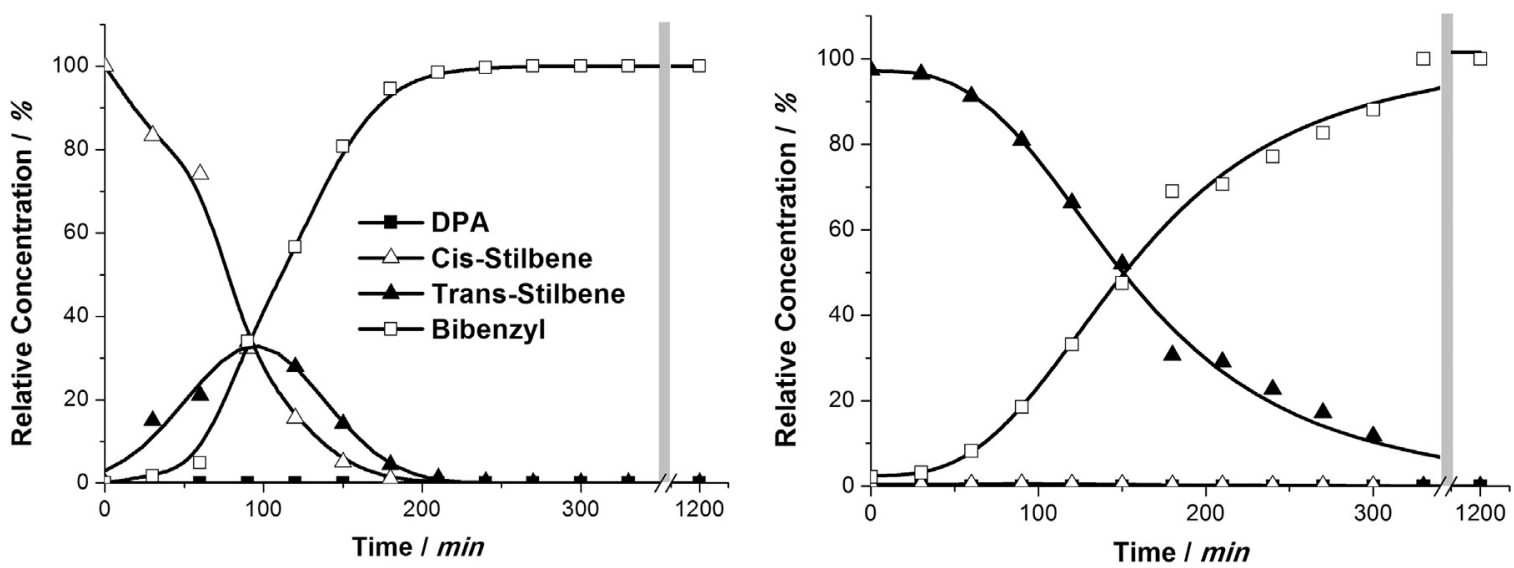

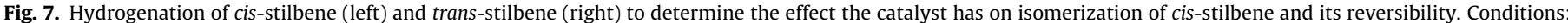
$150{ }^{\circ} \mathrm{C}, 100$ bar $_{2}$, cyclohexane solvent, $270 \mathrm{mg}$ catalyst ( $\left.1 \mathrm{mmol} \mathrm{NaAlH}_{4}\right), 5 \mathrm{mmol}$ cis-/trans-stilbene.

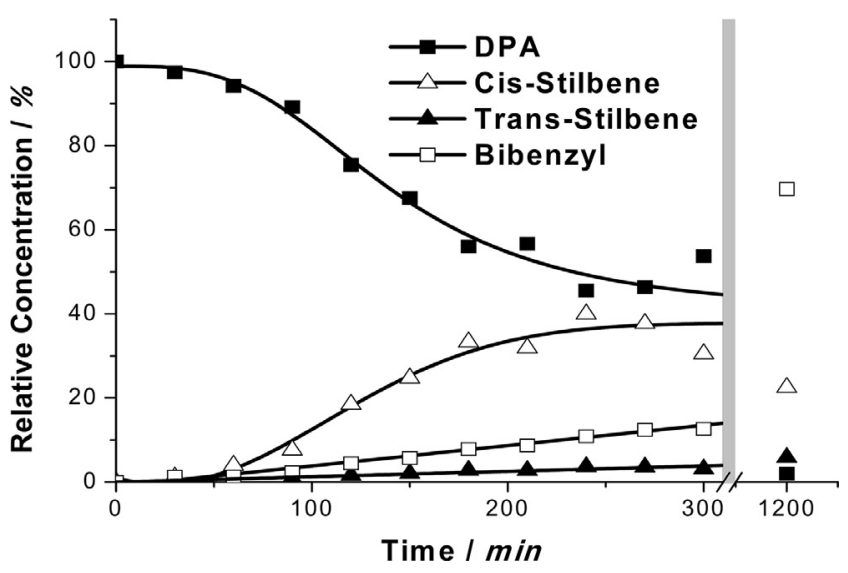

Fig. 8. Hydrogenation of DPA performed with a catalyst loading of $54 \mathrm{mg}(0.2 \mathrm{mmol}$ $\mathrm{NaAlH}_{4}$ ) and $5 \mathrm{mmol}$ DPA. $150^{\circ} \mathrm{C}, 100$ bar hydrogen, cyclohexane solvent.

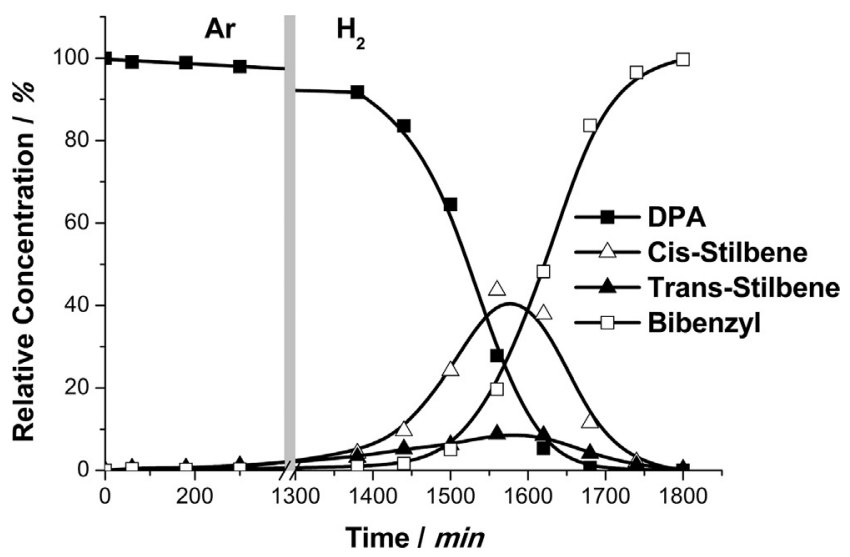

Fig. 9. Hydrogenation of DPA in the absence of gaseous hydrogen until $1400 \mathrm{~min}$ where hydrogen pressure was applied. The reaction was performed in cyclohexane at $150{ }^{\circ} \mathrm{C}$ with 80 bar Ar pressure initially before switching to 100 bar $\mathrm{H}_{2}$ pressure. A DPA: $\mathrm{NaAlH}_{4}$ molar ratio of 5:1 was used.

the catalyst was tested in the absence of gaseous hydrogen (Fig. 9). Hydrogenation occurs in the absence of gaseous hydrogen. This demonstrates that hydrogenation can proceed via extraction of hydrogen from the $\mathrm{NaAlH}_{4}$ lattice. However, the rate of hydrogenation is low; reaching less than $10 \%$ conversion of DPA in $24 \mathrm{~h}$, which corresponds to the consumption of $18 \%$ of the hydrogen in the catalyst. Once hydrogen pressure is introduced, the reaction proceeds much more rapidly to $100 \%$ conversion to bibenzyl (BB) within $6 \mathrm{~h}$. Possibly the extraction of hydrogen leads to a change in the nanostructure of the material (increasing the accessibility of the active sites) or an intrinsic change in the nature of the active sites.

Interestingly, if the hydrogen gas is introduced after a hydrogen-free period the catalyst is more selective toward cisstilbene than when hydrogen is introduced at the start of the reaction. At $50 \%$ conversion the cis:trans ratio is 5 , which is a fivefold increase compared to the reaction shown in Fig. 3. There are two possible explanations for this, the first being the aforementioned altered active surface area resulting from dehydrogenation of the catalyst. The other is that the intrinsic properties of the catalyst are changed during the Ar stage. However, in order to better understand this further investigation is required.

\section{Conclusions}

In conclusion, a transition-metal-free catalyst for hydrogenation of terminal and internal alkynes and alkenes has been demonstrated which consists of carbon supported $\mathrm{NaAlH}_{4}$ using a carbon aerogel type support. The catalyst has the ability to hydrogenate a number of substrates, including conjugated systems and unfunctionalized molecules. Activity depends on solvent due to competitive adsorption effects on the catalyst surface, as confirmed experimentally. Using cyclohexane as a solvent the activity for DPA hydrogenation is $1 \mathrm{~mol}$ (DPA) $\mathrm{mol}^{-1}\left(\mathrm{NaAlH}_{4}\right) \mathrm{h}^{-1}$ at $150{ }^{\circ} \mathrm{C}$. In contrast to typically used Pd catalysts the selectivity is 12 times higher in favor of the cis-stilbene intermediate at low catalyst loadings.

Additionally, we have shown that hydrogenation can occur via direct hydrogen extraction from the $\mathrm{NaAlH}_{4}$ lattice. When the catalyst is partially dehydrogenated before addition of hydrogen gas pressure, selectivity is 5 times higher toward cis-stilbene than when hydrogen pressure is applied at the beginning of the reaction. As this new type of catalyst is based solely on light and abundant elements and does not contain any transition metals, yet can still display high selectivity toward the cis-alkene, it promises a new avenue in the development of catalysts for selective hydrogenation.

\section{Author contributions}

The manuscript was written through contributions of all authors. All authors have given approval to the final version of the manuscript. 
The Project has been conceived by Petra de Jongh. Experimental work was carried out by Peter Bramwell, Jinbao Gao and Bernd de Waal. The major part of the text has been written by Peter Bramwell with significant contributions from Petra de Jongh, Krijn de Jong and Robertus Klein Gebbink.

\section{Funding sources}

This work was supported by the Netherlands Organization for Scientific Research [NWO-ECHO Grant No. 712.012.004].

\section{Notes}

The authors declare no competing financial interests.

\section{Acknowledgment}

The authors thank the financial support of the Netherlands Organisation for Scientific Research (NWO-ECHO grant). The authors would also like to thank Rien van Zwienen and Ad Mens for technical support and Dr. Michael Felderhoff of the MaxPlanck-Institut für Kohlenforschung for the stimulating discussions.

\section{Appendix A. Supplementary material}

Supplementary data associated with this article can be found, in the online version, at http://dx.doi.org/10.1016/j.jcat.2016.09.024.

\section{References}

[1] X. Cui, K. Burgess, Chem. Rev. 105 (2005) 3272-3296.

[2] D.-S. Wang, O.-A. Chen, S.-M. Lu, Y.-G. Zhou, Chem. Rev. 112 (2012) 2557 2590.

[3] J.-H. Xie, S.-F. Zhu, Q.-L. Zhou, Chem. Rev. 111 (2011) 1713-1760.

[4] P. Hauwert, G. Maestri, J.W. Sprengers, M. Catellani, C.J. Elsevier, Angew. Chem. 47 (2008) 3223-3226.
[5] J.W. Sprengers, J. Wassenaar, N.D. Clement, K.J. Cavell, C.J. Elsevier, Angew. Chem. 44 (2005) 2026-2029.

[6] M.W. van Laren, C.J. Elsevier, Angew. Chem. Int. Ed. 38 (1999) 3715-3717.

[7] P. Hauwert, R. Boerleider, S. Warsink, J.J. Weigand, C.J. Elsevier, J. Am. Chem. Soc. 132 (2010) 16900-16910.

[8] A.M. Kluwer, T.S. Koblenz, T. Jonischkeit, K. Woelk, C.J. Elsevier, J. Am. Chem. Soc. 127 (2005) 15470-15480.

[9] H. Lindlar, Helv. Chim. Acta 35 (1952) 446-450.

[10] E.V. Starodubtseva, M.G. Vinogradov, O.V. Turova, N.A. Bumagin, E.G. Rakov, V. I. Sokolov, Catal. Commun. 10 (2009) 1441-1442.

[11] D. Teschner, J. Borsodi, A. Wootsch, Z. Révay, M. Hävecker, A. Knop-Gericke, S. D. Jackson, R. Schlögl, Science 320 (2008) 86-89.

[12] M. García-Mota, B. Bridier, J. Pérez-Ramírez, N. López, J. Catal. 273 (2010) 92102.

[13] K. Chernichenko, A. Madarász, I. Pápai, M. Nieger, M. Leskelä, T. Repo, Nat. Chem. 5 (2013) 718-723.

[14] D.W. Stephan, G. Erker, Angew. Chem. Int. Ed. 49 (2010) 46-76.

[15] L.J. Hounjet, D.W. Stephan, Org. Process Res. Dev. 18 (2014) 385-391.

[16] G. Kreiselmeier, W. Frey, B. Föhlisch, Tetrahedron 62 (2006) 6029-6035.

[17] A.T. Tran, V.A. Huynh, E.M. Friz, S.K. Whitney, D.B. Cordes, Tetrahedron Lett. 50 (2009) 1817-1819.

[18] G. Streukens, F. Schüth, J. Alloys Compd. 474 (2009) 57-60.

[19] E.H. Majzoub, F. Zhou, V. Ozolinš, J. Phys. Chem. C 115 (2011) 2636.

[20] S. Zheng, Y. Li, F. Fang, G. Zhou, X. Yu, G. Chen, D. Sun, L. Ouyang, M. Zhu, J. Mater. Res. 25 (2010) 2047.

[21] J. Gao, P. Ngene, I. Lindemann, O. Gutfleisch, K.P. de Jong, P.E. de Jongh, J. Mater. Chem. 22 (2012) 13209.

[22] J. Gao, P. Adelhelm, M.H.W. Verkuijlen, C. Rongeat, M. Herrich, P.J.M. van Bentum, O. Gutfleisch, A.P.M. Kentgens, K.P. de Jong, P.E. de Jongh, J. Phys. Chem. C 114 (2010) 4675-4682.

[23] M.H.W. Verkuijlen, J. Gao, P. Adelhelm, P.J.M. van Bentum, P.E. de Jongh, A.P.M. Kentgens, J. Phys. Chem. C 114 (2010) 4683-4692.

[24] P. Adelhelm, K.P. de Jong, P.E. de Jongh, Chem. Commun. (2009) 6261-6263.

[25] Y.S. Au, M. Klein Obbink, S. Srinivasan, P.C.M.M. Magusin, K.P. de Jong, P.E. de Jongh, Adv. Funct. Mater. 24 (2014) 3604-3611.

[26] P. Ngene, P. Adelhelm, A.M. Beale, K.P. de Jong, P.E.J. de Jongh, Phys Chem. C 114 (2010) 6163-6168.

[27] P. Ngene, R. van den Berg, M.H.W. Verkuijlen, K.P. de Jong, P.E. de Jongh, Energy Environ. Sci. 4 (2011) 4108.

[28] B. Bogdanoviç, R.A. Brand, A. Marjanovic, M. Schwickardi, J. Tölle, J. Alloys Compd. 302 (2000) 36-58.

[29] S.A. Al-Muhtaseb, J.A. Ritter, Adv. Mater. 15 (2003) 101-114.

[30] T. Tomoya, S. Yoshiko, H. Toshihiko, I. Naotsugu, Fluid Phase Equilib. 228-229 (2005) 499-503.

[31] J.K. Rice, A.P. Baronavski, J. Phys. Chem. 96 (1992) 3359-3366. 